Questions vives

\section{Questions Vives}

Recherches en éducation

$N^{\circ} 29 \mid 2018$

La bienveillance en éducation : approches compréhensives et critiques

\title{
Management des établissements scolaires : l'appui sur l'intelligence émotionnelle et la bienveillance
}

caring management in education: support on emotional intelligence and caring

Lyne Bélanger et Gwénola Réto

\section{(2) OpenEdition}

\section{Journals}

Édition électronique

URL : https://journals.openedition.org/questionsvives/3342

DOI : 10.4000 /questionsvives.3342

ISSN : 1775-433X

Éditeur

Université Aix-Marseille (AMU)

Édition imprimée

ISBN : 978-2-912643-53-7

ISSN : 1635-4079

Référence électronique

Lyne Bélanger et Gwénola Réto, « Management des établissements scolaires : l'appui sur l'intelligence émotionnelle et la bienveillance », Questions Vives [En ligne], N²9 | 2018, mis en ligne le 19 décembre 2018, consulté le 08 décembre 2022. URL : http://journals.openedition.org/questionsvives/3342 ;

DOI : https://doi.org/10.4000/questionsvives.3342

Ce document a été généré automatiquement le 29 septembre 2020

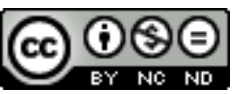

Creative Commons - Attribution - Pas d'Utilisation Commerciale - Pas de Modification 4.0 International - CC BY-NC-ND 4.0

https://creativecommons.org/licenses/by-nc-nd/4.0/ 


\title{
Management des établissements scolaires : l'appui sur l'intelligence émotionnelle et la bienveillance
}

\author{
Caring management in education: support on emotional intelligence and caring
}

\author{
Lyne Bélanger et Gwénola Réto
}

1 La Loi de refondation de l'École française (2013) donne une place forte à la bienveillance dans le cadre de son projet. Considérer la bienveillance comme « condition nécessaire à l'engagement et à la réussite» (Lapeyronnie, 2014) appelle le personnel enseignant à développer de nouvelles postures et pratiques, et invite à repenser la dimension collective du métier. Parallèlement, plusieurs rapports ou travaux de recherche révèlent la souffrance du corps enseignant, venant interroger leur bien-être au travail (Murat \& Simonis-Sueur, 2015) alors que, plus que jamais, on leur demande de se préoccuper davantage du bien-être de leurs élèves. Or une étude de l'OCDE (2008) reconnaît la place centrale que doivent occuper les directions d'établissements scolaires ${ }^{1}$ dans l'amélioration du système d'éducation. Manager ${ }^{2}$ le personnel scolaire nécessite de savoir prendre en compte non seulement leurs compétences, mais également tout un ensemble de ressources diversifiées : émotions, sentiments, rapport au travail, culture, maitrise du langage, confiance en soi, etc. (Le Boterf, 2010). Appelées à mettre en œuvre de nouvelles pratiques managériales, il y a lieu de s'interroger sur ce que les directions disent mettre en œuvre et sur l'intérêt qu'elles auraient à déployer un management bienveillant dans les organisations dont elles sont responsables. Cela permettrait de caractériser ce qui pourrait relever d'un tel management, notamment pour les établissements scolaires. S'appuyant sur deux thèses en éducation (Bélanger, 2017, au Québec, Réto, 2018a, en France), cet article propose de contribuer à cette interrogation en prenant appui sur ce que des personnels disent de l'exercice de leur responsabilité.

2 Dans sa thèse de doctorat, Bélanger (2017) montre l'importance de l'intelligence émotionnelle (IE) dans les pratiques de management en éducation, comme voie d'approfondissement et comme levier de développement pour le mieux-être d'un climat de travail. Les résultats de sa recherche proposent un modèle de compétences 
émotionnelles (CE) dont les dimensions conceptuelles rejoignent la bienveillance à travers la perception, l'évaluation et l'expression des émotions, leur compréhension et analyse, leur régulation réflexive, leur utilisation et l'attention qui leur est portée. Cette dernière dimension prend appui sur la relation de caring, notion proche de la bienveillance, ce que nous préciserons. De son côté, Réto (2018a) propose une clarification des dimensions constitutives de la bienveillance, mettant en évidence ses dimensions intentionnelle, interactionnelle, attentionnelle et affective, dimensions qui seront précisées ultérieurement.

3 Prenant appui sur une analyse secondaire des données, cet article vise à interroger le rapport entre la bienveillance et l'intelligence émotionnelle dans les pratiques déclarées par les directions des établissements scolaires, et, ce faisant, à caractériser ce qui relèverait d'un management bienveillant. Partant du constat de l'essor de l'appel à ce mode de management, nous présentons les préoccupations sociales et scientifiques concernant le management des établissements scolaires. La deuxième partie est dédiée aux cadres de référence mobilisés pour interpréter les données et la suivante présente le cadre méthodologique adopté. Enfin, la présentation des résultats des deux études permet d'envisager les apports de la prise en compte de l'intelligence émotionnelle comme levier managérial et d'ouvrir la discussion sur le management bienveillant en milieu d'éducation.

\section{Le management des établissements scolaires, une problématique internationale}

4 La notion de management bienveillant, apparue depuis une dizaine d'années, tend à s'imposer comme voie de renouvellement des pratiques managériales. Nous présentons quelques caractéristiques de cette émergence avant de relever les points de tension liés au management des établissements scolaires partagés à l'international et les spécificités inhérentes à nos terrains de recherche : le Québec et la France.

\subsection{Le « management bienveillant », un modèle en discussion}

$5 \quad$ Le défi des managers contemporains est de devoir piloter des collectifs dans un monde complexe sur lequel règnent des exigences de productivité, de compétitivité et d'urgence qui demandent de faire preuve sans cesse de mobilité, de réactivité, d'efficacité, enjoignant à une responsabilité toujours plus grande (Gaulejac, 2004). Différents travaux consacrés à l'hypermodernité mettent ainsi en avant l'affaiblissement du sens (Ehrenberg, 1998), la mutation du rapport au temps (Aubert, 2003) et l'altération relationnelle qui engendrent une difficulté à ressentir le monde comme cohérent. La complexité qui caractérise notre période contemporaine (Morin, 2005) appelle à de nouvelles manières d'appréhender le monde et les événements. C'est dans ce contexte sous pression que la notion de bienveillance se répand progressivement en entreprise, notamment dans le champ du management. Sur le plan international, elle s'appuie sur les théories du care. Ainsi, en 2010, le congrès annuel de l'Academy of Management a été consacré au Dare to Care (traduisible par « Osez la bienveillance »). Il s'inscrivait dans un mouvement donnant une place forte aux valeurs, au souci de l'autre et à la sollicitude, visant à redonner du sens au travail et à en envisager une organisation plus respectueuse, cherchant à accroître le bien-être au travail. En 2012, l'Academy of Management a aussi 
publié un numéro spécial consacré à la compréhension et à la création d'organisations bienveillantes (Rynes, Bartunek, Dutton \& Margolis, 2012). Conjuguant les notions de care et de compassion, une vision plus humaniste du management est proposée, considérant que la mise en œuvre de certaines valeurs en contexte de travail et la prise en compte des chaînes d'interdépendance existantes permettent la productivité. Mais Linhart (2015) dénonce cette tendance «surhumanisante » dans laquelle elle voit une véritable aubaine pour créer du consensus et sortir du registre de la confrontation. Pour elle, il s'agirait d'un management conduisant à "une déprofessionnalisation systématique des travailleurs par un management soucieux avant tout de les contrôler et de maîtriser leur travail» (p. 12). Management et bienveillance seraient donc des notions contradictoires. Les données seront alors à interroger au regard de ces positions, entre fascination et suspicion, et du contexte spécifique de nos recherches, les établissements scolaires.

\subsection{Le management des établissements scolaires au cœur des préoccupations}

\section{Des préoccupations internationalement partagées}

Les préoccupations de bien-être et de qualité de vie qui ont été à l'origine de l'essor du management bienveillant se retrouvent dans les établissements scolaires (Réto, 2018b). La direction occupe une fonction stratégique puisqu'elle est considérée comme jouant un rôle clé dans l'amélioration des systèmes éducatifs. Selon l'OCDE (2008), le monde de l'éducation se questionne sur la réalité des compétences nécessaires au personnel d'encadrement des établissements d'enseignement pour assurer un exercice efficace de leurs fonctions et répondre aux nouveaux défis du XXIe siècle. Elle préconise d'offrir un soutien à celles et ceux qui exercent la fonction de direction. Le rapport de 2008 reconnait notamment la place que doivent occuper les directions dans l'amélioration des résultats en influant «sur les motivations, les capacités et les conditions de travail du personnel enseignant, qui elles-mêmes influent sur les pratiques pédagogiques et l'apprentissage des élèves » (p. 37). Par conséquent, l'OCDE (2008) craint que la mission de la direction « conçue pour l'ère industrielle n'ait pas connu une évolution suffisante permettant de résoudre les problèmes complexes qui se posent aux écoles du $21^{\mathrm{e}}$ siècle » (p. 16). Au-delà des prescriptions de l'OCDE dont la perspective est surtout économique apparaît le souci d'une perspective éducative plus humaniste (Vienneau, 2005). En même temps, la fonction de direction a connu des changements importants ces dernières décennies dans de nombreux pays. Endrizzi et Thibert (2012) indiquent que « de quelques responsabilités supplémentaires assumées par un enseignant, elle est devenue [...] une fonction à part entière »(p.9). Dutercq, Gather Thurler et Pelletier (2015) soulignent d'ailleurs les évolutions en matière de régulation et d'encadrement, et des pratiques de pilotage qui s'inscrivent dans une tradition très bureaucratique. Pourtant, l'élargissement des rôles et des responsabilités nécessite des compétences spécifiques, notamment interpersonnelles (OCDE, 2012).

\section{Préoccupations au Québec (Canada)}

7 Pour répondre à ces défis, le monde de l'éducation nord-américain a amorcé une ère de changement sur la réflexion des compétences nécessaires aux directions. Le réseau des établissements collégiaux ${ }^{3}$ du secteur public est confronté à ces enjeux, comme les autres 
établissements scolaires du Québec. La charge de responsabilités qui incombe à la direction est lourde. En effet, sous l'autorité d'un conseil d'administration, la direction générale assume la responsabilité totale de la gestion des activités éducatives, administratives et financières de même que des ressources humaines et matérielles. De plus, elle entretient et développe des liens étroits avec les partenaires du milieu et de l'institution, afin d'assurer le développement de l'institution et contribuer au développement socioéconomique de sa région administrative. En fait, cette fonction de travail de dirigeants de haut niveau oblige à de fortes compétences professionnelles qui se traduisent par l'appropriation de savoirs, savoir-faire et savoir-être. Ce type d'emploi requiert des aptitudes en relations humaines: bonnes relations de travail, capacité d'écoute, tact et diplomatie, empathie, pouvoir de persuasion, habiletés à négocier, à faire travailler des collaborateurs, à motiver et à diriger des équipes (Gouvernement du Québec, 2013).

8 Ainsi, dans son étude, Poirel (2012) soutient que «Le travail des directions d'école est devenu extrêmement exigeant sur le plan émotionnel» (p.32). Mintzberg (2014) mentionne les pressions continuelles vécues par les directions, dont les cadences effrénées, les interruptions, le désordre à remettre en ordre et l'ambiguïté de l'action. Il cite aussi le dilemme de la délégation, le syndrome de la superficialité et le conflit de la confiance qui deviennent des paradoxes inéluctables du management. Pour lui, le management est une pratique et non une profession parce que ce que font les managers ${ }^{4}$ ou ce qu'ils ne font pas diffèrent d'un contexte à l'autre. Ainsi, le management est avant tout considéré selon ce que les managers font en se situant, c'est-à-dire la manière dont ils gèrent la situation en tenant compte du contexte de réalisation, dans leurs pratiques quotidiennes (Mintzberg, 2010).

9 La difficulté de l'exercice professionnel des directions est mise en évidence par différents travaux. Certaines études empiriques portent sur les préoccupations exprimées quant à leur importante charge de travail, la complexification de leur tâche, l'augmentation des compétences multidisciplinaires nécessaires, et les répercussions sur leur état de santé et sur celui de leur organisation (Bélanger, 2016; Garon, Théorêt, Hrimech \& Carpentier, 2006; OCDE, 2008). L'émergence de ces problématiques organisationnelles a suscité l'intérêt de chercheurs multidisciplinaires qui s'interrogent sur la place de l'IE en éducation (Lafranchise, Lafortune \& Rousseau, 2013 ; Letor, 2006 et Pharand \& Doucet, 2013). Par conséquent, Bélanger (2017) a voulu comprendre comment les directions générales du réseau collégial exercent leurs $\mathrm{CE}$ en situation professionnelle.

\section{Préoccupations en France}

10 Parallèlement aux préoccupations québécoises, la France a connu une importante réforme de son système éducatif qui s'est exprimée par la Loi de la refondation de l'École française (MEN, 2013). Les enquêtes nationales comme internationales (OCDE, 2014; DEPP, 2011) avaient révélé des constats de tension et d'inégalités inquiétants. Le développement des travaux sur le climat scolaire montre un climat dégradé (Debarbieux, 2015) qui explique le souhait de mieux vivre à l'école. Cette réforme a donc donné une place jusqu'ici inédite au bien-être, à relier à un attendu de bienveillance pour l'école dans son ensemble (Réto, 2018b). La nouvelle loi stipule que « les conditions d'un climat scolaire serein doivent être instaurées dans les écoles et les établissements scolaires pour favoriser les apprentissages, le bien-être et l'épanouissement des élèves et de bonnes 
conditions de travail pour tous » (MEN, 2013). Il s'agit donc d'une priorité de politique publique pour l'éducation.

Dans la revue de question consacrée à la qualité de vie du personnel enseignant en relation avec celle des élèves, Rascle, Bergugnat, Florin et Guimard (2016) s'interrogent sur la manière de construire des communautés éducatives permettant de produire une certaine qualité de vie à l'école. Ils montrent qu'elle dépend de la qualité du climat scolaire et que la qualité de vie des enseignantes et enseignants est dépendante de leurs interactions avec les élèves et du management des établissements scolaires. Les liens entre bienveillance et qualité de vie à l'école sont étroits : l'appui sur une analyse des occurrences de la bienveillance dans les différents textes institutionnels publiés par le ministère de l'Éducation nationale français de 2013 à 2015 montre qu'elle est devenue un attendu pour l'institution dans son ensemble, en lien avec ces préoccupations du climat scolaire et du bien-être à l'École. La bienveillance constitue également un attendu relationnel structurant pour le personnel enseignant. Mais une recension des écrits démontre que la notion n'est pas stabilisée, ce qui a conduit Réto à vouloir comprendre comment se manifeste la bienveillance sur le terrain professionnel de l'enseignement à l'école française, et plus particulièrement à déterminer les conditions nécessaires à l'actualisation de la bienveillance à l'échelle de l'établissement.

Ferrière, Florin et Guimard (2016) montrent la place centrale des directions dans le cadre des interactions entre les personnels pour la qualité de vie dans les établissements scolaires. Les auteurs recommandent d'ailleurs de s'intéresser aux caractéristiques des directions du fait du rôle important qu'ils ont à jouer dans l'animation des collectifs dont ils portent la responsabilité. Ils soulignent également l'importance de la composante émotionnelle dans la relation éducative et invitent à la mise en œuvre de modalités de travail de gestion et de régulations émotionnelles dans les établissements scolaires. Dutercq et Mons (2015), quant à eux, soulignent la forte responsabilisation des directions, délicate à assumer d'autant que leur culture professionnelle s'appuie sur « un mélange de tradition pédagogique, de compétences administratives et d'aspirations managériales » (p. 22).

13 Ainsi, tant sur le plan international que national, le management des établissements scolaires pose question et constitue un réel défi. C'est pour contribuer à cette réflexion que nous avons décidé de mettre en dialogue nos deux recherches. Leur confrontation vise à permettre d'identifier quelques spécificités liées au management des établissements scolaires par les directions. Ainsi, nous interrogerons la manière dont la prise en compte de l'IE dans les pratiques managériales peut constituer un apport pour le management bienveillant en milieu d'éducation, notions qu'il convient de mieux définir avant de les discuter.

\section{Cadre de référence : définition des concepts}

Notre réflexion prend appui sur les cadres de référence spécifiques à nos recherches : le care, la sollicitude et la bienveillance pour Réto ; l'intelligence émotionnelle (IE) et les compétences émotionnelles (CE) et le « savoir agir avec compétence » pour Bellanger. 


\subsection{Care, sollicitude et bienveillance} deux s'enracinent dans le souci de l'autre, dans une prise en considération de la fragilité, de la vulnérabilité, et dans une pratique relationnelle. Le nom care renvoie au soin et à l'attention. Le terme de sollicitude lui est parfois substitué en français. Historiquement, celle-ci est rattachée au soin empreint de préoccupation et d'inquiétude, à une attention engendrant un trouble. Toutefois, sollicitude et bienveillance sont des termes proches. Issue du latin bene volens, la bienveillance désigne originellement un sentiment par lequel on veut du bien à quelqu'un. Foulquié et Saint-Jean (1969) lui identifient un sens fort et un sens faible : au sens fort, elle désigne la volonté réelle et efficace du bien d'autrui ; au sens faible, elle est une disposition favorable à l'égard d'autrui. En éducation, l'intentionnalité inhérente à l'exercice de la responsabilité éducative explique sans doute pourquoi le terme de bienveillance est préféré à celui de sollicitude, que l'on retrouve davantage dans le champ de la santé, associé à la notion de bientraitance.

dimension essentielle de la vie morale est celle de l'intersubjectivité. Mais pour Brugère (2011), au-delà d'une éthique, le care s'exerce et « appelle une activité d'accompagnement en vue du développement, du maintien ou de la restauration d'une puissance d'être, de dire ou d'agir » (p. 84). Convoquer l'éthique du care dans le cadre de la réflexion sur la responsabilité managériale invite à un déplacement: pour Laugier et Paperman (2008), « il y a bien un raisonnement spécifique du care : il ne valide pas les réponses en référence à des principes mais donne sens aux détails concrets, spécifiques et les rend intelligibles dans les contextes de vie des personnes » (p. XX). Zielinski (2011) souligne d'ailleurs qu'il s'agit de porter un care qui ne soit pas seulement sous-tendu par la peur de nuire à autrui mais "qui se conforme à une "éthique du bien-être d'autrui" fondée sur l'interdépendance entre soi et autrui, la réussite à faire coïncider sens des responsabilités et attention aux autres » (p. 23). La relation de care implique donc un art de la relation qui ouvre la voie au développement de compétences spécifiques, et une éthique fondée sur des relations prises dans une chaîne de vulnérabilité et de dignité, d'interdépendance et de réciprocité.

d'une recension des écrits en philosophie et en sciences de l'éducation, Réto (2018a) propose de définir la bienveillance en prenant appui sur quatre dimensions interreliées qui sont ici spécifiées au regard de la pratique managériale en éducation. En premier lieu, la bienveillance peut se définir par sa dimension intentionnelle (1). Pour la direction, l'intentionnalité est guidée et cadrée par la mission confiée, et le bien recherché est prescrit institutionnellement: c'est le bien d'une communauté éducative qui a pour visée le développement éducatif et scolaire des élèves qui lui sont confiés. La volonté et le discernement $\mathrm{du}$ bien s'appuient sur la connaissance du cadre et l'intériorisation des valeurs et des normes, notamment professionnelles. Définir la bienveillance en éducation scolaire pousse ainsi à l'inscrire dans le champ de l'éthique professionnelle en lien avec l'assignation à responsabilité (Prairat, 2012). La bienveillance comporte aussi une dimension interactionnelle (2). Dans la relation éducative, il s'agit d'une relation «située ", où chacun occupe une place précise. Cette assignation de place est doublée ici par la dimension managériale, la direction représentant symboliquement le pouvoir et l'autorité institutionnelle. En tension entre vouloir et pouvoir, la relation 
bienveillante nécessite que la direction soit capable d'adopter une juste distance, différente selon les publics auxquels elle s'adresse (personnels de l'établissement, élèves, familles, partenaires de l'école, etc.), ce qui constitue un défi. La bienveillance comporte également une dimension affective (3), inhérente à la relation. Reconnaître cette composante affective permet de définir une place pour la prise en compte professionnelle des affects et des émotions. Nous ne développons pas davantage cette dimension reprise par les travaux de Bélanger (2017). Enfin, la bienveillance comporte une dimension attentionnelle (4): elle nécessite l'attention à l'autre, la disponibilité et la capacité de veille et de vigilance inhérentes aussi à la responsabilité.

Inscrire la réflexion sur le management des établissements scolaires en lien avec le care et la bienveillance implique ainsi de donner une place à la prise en compte des situations professionnelles spécifiques ainsi que la prise en compte des émotions par les directions ${ }^{5}$.

\subsection{Interrelation de l'intelligence émotionnelle et des compétences émotionnelles}

La thèse de Bélanger (2017) propose de réfléchir à l'interrelation entre l'IE et les CE. À l'instar de Kotsou $(2012,2016)$ et Mikolajczak, Quoidbach, Kotsou et Nélis (2014), c'est le modèle théorique de Mayer et Salovey (1997) sur l'IE qui a été retenu dans sa recherche puisqu'il a ouvert le champ à de nombreuses recherches pluridisciplinaires dans le domaine des émotions. Mayer et Salovey (1997) proposent une nouvelle définition de l'IE, toujours présente en recherche :

Emotional intelligence involves the ability to perceive accurately, appraise, and express emotion; the ability to access and/or generate feelings when they facilitate thought; the ability to understand emotion and emotionnel knowledge; and the ability to regulate emotions to promote emotionnel and intellectual growth ${ }^{6}$ (p. 10).

Le modèle de l'IE est fondé sur des habiletés qui amènent la personne à développer des $\mathrm{CE}$ en contexte professionnel ${ }^{7}$, car le rôle crucial des $\mathrm{CE}$ se situe au niveau de l'adaptation à la situation professionnelle. Ce modèle semble opérationnalisable dans une organisation puisqu'il informe des compétences à développer selon la situation professionnelle à gérer. En fait, l'IE est un ensemble d'habiletés intrapersonnelles et interpersonnelles qui permet à la personne d'exercer ses $\mathrm{CE}$ en les adaptant au contexte professionnel. L'IE constitue ainsi l'un des paramètres importants de la performance et du bien-être puisque, dans certains cas, les qualités émotionnelles explicitent davantage ces paramètres que les compétences cognitives (Kotsou, 2012, 2016). Prenant appui sur les travaux de Kotsou $(2012,2016)$ et de Salovey et Mayer (1990), Mikolajczak et al. (2014) qui ont élaboré un modèle de CE. Celui-ci renvoie aux trois groupes de compétences des fondateurs (Salovey \& Mayer, 1990) : les CE tournées vers soi, les CE tournées vers les autres, les CE tournées vers l'action. Le tableau 1 présente les cinq compétences de base ainsi que les habiletés émotionnelles intrapersonnelles et interpersonnelles nécessaires à l'opérationnalisation des CE, issues du modèle de l'IE de Mayer et Salovey (1997) et du modèle de Mikolajczak et al. (2014), intéressant par sa visée managériale et le lien avec le concept de l'IE de Salovey et Mayer (1990).

Tableau 1 : Les cinq compétences émotionnelles de base

Les habiletés issues de l'intelligence émotionnelle 


\begin{tabular}{|c|c|c|}
\hline $\begin{array}{l}\text { Identification des } \\
\text { compétences } \\
\text { émotionnelles }\end{array}$ & $\begin{array}{l}\text { Habiletés } \\
\text { intrapersonnelles }\end{array}$ & $\begin{array}{ll}\text { Habiletés émotionnelles } \\
\text { interpersonnelles }\end{array}$ \\
\hline $\begin{array}{l}\text { 1. Identifier les } \\
\text { émotions chez soi et } \\
\text { chez les autres }\end{array}$ & Identifier son vécu émotionnel & $\begin{array}{l}\text { Identifier les processus } \\
\text { émotionnels des autres }\end{array}$ \\
\hline $\begin{array}{l}2 . \quad \text { Exprimer } r \text { ses } \\
\text { émotions et faciliter } \\
\text { l'expression } \\
\text { autres }\end{array}$ & $\begin{array}{l}\text { Exprimer ses émotions de manière } \\
\text { adaptée au contexte }\end{array}$ & $\begin{array}{l}\text { Permettre aux autres d'exprimer } \\
\text { leurs sentiments et faciliter cette } \\
\text { expression }\end{array}$ \\
\hline $\begin{array}{l}\text { 3. Comprendre son } \\
\text { vécu émotionnel et } \\
\text { celui des autres }\end{array}$ & $\begin{array}{l}\text { Comprendre son vécu émotionnel } \\
\text { dans le contexte présent }\end{array}$ & $\begin{array}{l}\text { Comprendre le vécu et les } \\
\text { réactions de ses interlocuteurs }\end{array}$ \\
\hline $\begin{array}{lr}4 . \quad \text { Réguler } & \text { les } \\
\text { émotions au } & \text { niveau } \\
\text { personnel } & \text { et } \\
\text { relationnel } & \end{array}$ & $\begin{array}{l}\text { Gérer et réguler ses émotions } \\
\text { désagréables en fonction du contexte } \\
\text { et de ses objectifs } \\
\text { Réguler ses émotions positives et } \\
\text { faire de ses émotions un point fort: } \\
\text { utiliser ses émotions pour être plus } \\
\text { créatif, prendre de meilleures } \\
\text { décisions }\end{array}$ & $\begin{array}{l}\text { Réguler et gérer les émotions } \\
\text { désagréables dans leur dimension } \\
\text { relationnelle, par exemple dans } \\
\text { le cadre d'un conflit } \\
\text { Réguler les émotions positives } \\
\text { dans la relation, par exemple } \\
\text { pour créer de la motivation, } \\
\text { installer une ambiance créative }\end{array}$ \\
\hline $\begin{array}{lr}5 . \quad \text { Utiliser } & \text { les } \\
\text { émotions au } & \text { niveau } \\
\text { personnel } & \text { et } \\
\text { relationnel } & \end{array}$ & $\begin{array}{l}\text { Utiliser ses émotions pour accroître } \\
\text { leur efficacité (au niveau de la } \\
\text { réflexion, des décisions, des actions) }\end{array}$ & $\begin{array}{l}\text { Utiliser les émotions des autres } \\
\text { pour accroître leur efficacité (au } \\
\text { niveau de la réflexion, des } \\
\text { décisions, des actions) }\end{array}$ \\
\hline
\end{tabular}

Synthèse provenant des modèles de Kotsou $(2012,2016)$ et Mikolajczak et al. (2014)

Dans la littérature, l'idée que «les compétences émotionnelles réfèrent aux différences dans la manière dont les individus identifient, expriment, comprennent, utilisent et régulent leurs émotions et celles d'autrui » (Mikolajczak et al., 2014) semble faire consensus. Le concept de l'IE a été clarifié par plusieurs auteurs, cependant nous optons pour la définition de Poirel (2012) qui émerge d'une étude auprès de directions en administration de l'éducation au Québec:

Les compétences émotionnelles s'inscrivent dans un savoir agir complexe constitué d'un ensemble d'habiletés (verbales et non verbales) qui permettent à un individu de générer, reconnaître, exprimer, comprendre et évaluer ses propres émotions et celles des autres, dans le contexte d'une situation professionnelle concrète, de manière à orienter les pensées et les actions permettant d'affronter efficacement les exigences et les pressions de l'environnement en vue d'atteindre un résultat (p. 32).

Le construit théorique des cinq $\mathrm{CE}$ de base (tableau 1) s'applique bien au paradigme de gestion de l'agir compétent (Le Boterf, 2013) dans lequel l'IE est considérée comme ressource personnelle. Il permet aussi de continuer à clarifier le lien entre compétence et situation et sera éclairant pour comprendre les spécificités managériales qui mobilisent les $\mathrm{CE}$ selon le contexte situé. 


\subsection{Un modèle d'action, le « savoir agir avec compétence »} introduit une rupture évolutive dans la manière de raisonner la compétence. Il propose une évolution intéressante par sa vision de l'intégration des compétences dans un processus dynamique. Le concept de compétence se définit habituellement comme « une somme de savoirs, de savoir-faire et de savoir-être » (Le Boterf, 2010, p. 15). Pourtant, la compétence n'est pas une addition de ressources. La considérer ainsi, « c'est raisonner en termes d'assemblage et non pas de combinatoire» (p.17), omettant la dynamique interactionnelle qui existe entre ces éléments. Le modèle du «savoir agir avec compétence " privilégie l'approche situationnelle et conceptualise la manière dont les compétences individuelles et collectives peuvent se construire. En ce sens, la définition de la compétence de Tardif, Fortier et Préfontaine (2006) témoigne de la même perspective épistémologique: "Un savoir-agir complexe prenant appui sur la mobilisation et la combinaison efficaces d'une variété de ressources internes et externes à l'intérieur d'une famille de situations» (p.22). La situation apparaît à une personne telle qu'elle l'appréhende, la détecte, la problématise, selon ce qu'elle envisage en vertu de l'action à préconiser pour arriver à un résultat escompté. Ce processus situationnel diffère selon l'action en cours et s'opère par l'intermédiaire d'un faisceau de ressources très diversifiées et personnalisées d'un individu à l'autre, dans une visée d'adaptabilité. Le savoir-être mentionné dans la somme de ressources est une notion importante mais ambiguë car « elle fait référence à des comportements professionnels qui doivent être mis en œuvre dans des situations de travail » (Le Boterf, 2010, p. 18).

Le Boterf (2010) propose une modélisation du processus qu'une personne, en l'occurrence une direction, met en œuvre pour agir avec pertinence et compétence en situation professionnelle. Dans ce modèle systémique, le savoir-faire relationnel et les ressources émotionnelles font partie des ressources personnelles dont la direction a besoin lors des interactions avec le personnel, et pour la qualité des relations qu'il entretient avec eux (2010). Celles-ci se traduisent par des habiletés ou capacités de l'individu, lui permettant d'agir à bon escient mais aussi d'anticiper et de pressentir avec compétence une situation professionnelle. C'est la raison pour laquelle le modèle du « savoir agir avec compétence " a été retenu par Bélanger (2017) qui s'intéresse au rapport qu'entretient le personnel de direction générale à l'IE et son influence sur la qualité des relations avec son personnel.

\section{Cadre méthodologique des deux études : Québec et France}

Cet article s'appuyant sur l'analyse secondaire de données émanant de nos deux recherches distinctes. Nous présentons brièvement le cadre méthodologique retenu pour chacune de ces études effectuées auprès d'établissements au Québec et en France ${ }^{8}$. Si les deux ont adopté une méthodologie qualitative (Paillé \& Mucchielli, 2013) avec une posture compréhensive, chacune comporte des spécificités. 


\subsection{L'étude de Bélanger}

\section{Contexte et participants}

Pour la recherche effectuée au Québec, huit entretiens d'explicitation (Vermersch, 2014) ont été réalisés auprès de directions générales ${ }^{9}$ répertoriées dans huit collèges d'enseignement général et professionnel (cégeps) provenant de six régions administratives. Le tableau 2 propose un bref portrait des sujets et de leurs établissements.

Tableau 2 : Portrait des sujets et leurs milieux

\begin{tabular}{|l|l|l|l|l|l|}
\hline $\begin{array}{l}\text { Prénom } \\
\text { fictif }\end{array}$ & Sexe & Âge & Formation initiale & $\begin{array}{l}\text { Classification } \\
\text { cégep }{ }^{10}\end{array}$ & $\begin{array}{l}\text { Élèves temps- } \\
\text { plein }\end{array}$ \\
\hline Laurent & H & 60 & Gestion de l'éducation & Urbain & 1507 \\
\hline Benoît & H & 58 & Gestion des services sociaux & Rural & 2588 \\
\hline Marie & F & 51 & $\begin{array}{l}\text { Gestion en développement des } \\
\text { organisations }\end{array}$ & Urbain & 2530 \\
\hline Caroline & F & 49 & $\begin{array}{l}\text { Gestion financière } \\
\text { comptabilité }\end{array}$ & Rural & 1691 \\
\hline Marguerite & F & 43 & Langue et linguistique & Urbain & 3162 \\
\hline Anne & F & 58 & Psychopédagogie et andragogie & Urbain & 6448 \\
\hline Sophie & F & 55 & Science politique & Urbain & 5824 \\
\hline Fabien & H & 58 & Gestion de l'éducation & Urbain & 10863 \\
\hline
\end{tabular}

\section{Dispositif méthodologique}

L'opérationnalisation méthodologique de l'étude a permis de créer le récit d'explicitation de pratique professionnelle (REdEPP) comme méthode de collecte de données pour cette démarche de recherche exploratoire à perspective ethnosociologique. Le REdEPP est une méthode biographique professionnelle qui se distingue par deux fonctions méthodologiques : comme méthode de collecte de données appuyée par la technique de l'entretien d'explicitation (EdE), (Vermersch, 2014); comme méthode d'analyse qualitative en mode écriture. Enfin, ce nouveau dispositif méthodologique émerge de la filiation du récit de pratique (Bertaux, 2010), approche biographique dont la particularité suppose d'analyser et de comprendre les situations à partir du vécu des individus (Wacheux, 1996). De plus, la complémentarité apportée par la technique d'EdE permet de définir une conduite d'entretien visant la verbalisation de l'action (Vermersch, 2014). La conjugaison de deux outils méthodologiques menant à l'innovation du REdEPP oblige à une réflexion sur la congruence d'une démarche d'analyse tenant compte de la complexité des données à analyser. Cette analyse s'est effectuée progressivement vers 
une théorisation ancrée (Paillé \& Mucchielli, 2013). Paillé (1994) mentionne «qu'une théorie ancrée est construite et validée simultanément par la comparaison constante entre la réalité observée et l'analyse en émergence » (p. 150). Le logiciel QDA Miner a été utilisé pour faciliter la gestion des données et a permis d'accomplir avec rigueur cette analyse selon deux approches qualitatives : 1) l'analyse en mode écriture ; 2) l'analyse transversale thématique des récits validés.

\subsection{L'étude de Réto}

\section{Contexte et participants}

Pour la recherche effectuée en France, quatre entretiens semi-dirigés ont été réalisés auprès de directions de collèges ${ }^{11}$ d'une même région. Le tableau 3 propose un bref portrait des sujets et de leurs établissements.

Tableau 3 : Portrait des sujets et leurs milieux

\begin{tabular}{|l|l|l|l|l|l|}
\hline Désignation & Sexe & Âge & Formation initiale & Classification & Élèves \\
\hline Dir1 & H & 56 & $\begin{array}{l}\text { Sciences physiques } \\
\text { Sciences de l'éducation }\end{array}$ & Urbain & 925 \\
\hline Dir2 & F & 56 & $\begin{array}{l}\text { Lettres } \\
\text { Cadre de direction }\end{array}$ & Rural & 670 \\
\hline Dir3 & F & 46 & $\begin{array}{l}\text { Allemand } \\
\text { Cadre de direction }\end{array}$ & Urbain & 420 \\
\hline Dir4 & H & 59 & Management & Rural & 624 \\
\hline
\end{tabular}

\section{Dispositif méthodologique}

Un axe du guide d'entrevue portait sur l'actualisation de la bienveillance dans les pratiques professionnelles. Nous nous limiterons à cet axe dans le cadre de cet article. L'analyse des données s'appuie sur une démarche de thématisation (Paillé \& Mucchielli, 2013) en deux volets : (1) une thématisation continue ouverte qui a permis de construire un premier niveau d'analyse et a débouché sur l'identification de trois formes de bienveillance et d'une posture et de pratiques spécifiques ; (2) le recours au logiciel QDA Miner qui a été utilisé pour faciliter la gestion des données et permettre de confirmer la saillance thématique et d'ouvrir aux catégories conceptualisantes (Paillé \& Mucchielli, 2013).

\section{Résultats des recherches}

Avant de mettre en discussion les deux recherches, nous présentons les résultats sur lesquels nous reviendrons dans la mise en dialogue de nos travaux. 


\subsection{L'actualisation de la bienveillance selon les directions des établissements scolaires}

31 Au cours des entrevues, les directions ont peu discuté l'injonction à la bienveillance faite au système scolaire et à ses acteurs. Ils ont présenté cet appel comme logique et bienvenue. Ils ont d'ailleurs manifesté la conviction de la mettre en œuvre dans leurs pratiques managériales ${ }^{12}$ et se sont employés à documenter cet aspect, ce que nous présenterons ici.

\section{Les formes d'expression de la bienveillance dans les pratiques managériales}

32 Les résultats généraux de l'étude de Réto (2018a) permettent de dégager trois formes de conception et de mise en œuvre de la bienveillance dans les pratiques d'enseignement qui se retrouvent dans l'analyse des conceptions et pratiques managériales déclarées par les directions interrogées.

Une première forme permet d'envisager la bienveillance comme qualité personnelle (1). S'agissant des directions, elles manifestent dans leurs propos un goût pour la relation, une conception humaniste de la mission éducative, et la sensibilité au bien commun. La Dir2 exprime toutefois la nécessité de "sacrifier la bienveillance personnelle » dans l'exercice de la responsabilité de direction, au profit d'une autre forme de bienveillance reliée à l'incarnation du cadre nécessaire au déploiement de l'action pédagogique et éducative (2). Cette deuxième forme s'appuie sur la conscience de l'importance du rôle confié, notamment l'incarnation de l'autorité et la représentation de l'État. La mission de service apparaît pour certaines directions comme «transcendantale» (Dir2), pouvant aller jusqu'à une position sacrificielle (Dir4). En acceptant la responsabilité du développement et du pilotage de l'établissement, les directions se disent en devoir de garantir les cadres de l'action individuelle comme collective. C'est là le cœur des préoccupations managériales exprimées. Par ailleurs, les déclarations des directions sur leurs pratiques s'expriment dans une forme d'art managérial (3), art étant entendu comme l'ensemble de moyens et de procédés mis en œuvre pour manager dans cette perspective, mais aussi comme habilité et talent. Cette forme s'appuie sur une gamme de pratiques soutenant la communication, la structuration des relations et l'arbitrage des conflits, tout en visant l'optimisation des conditions de travail. Elle a des liens étroits avec le « prendre soin » et l'éthique du care.

\section{Le « prendre soin » et l'éthique du care}

Les directions disent accorder une place importante au "prendre soin » des différents membres de la communauté éducative (élèves, familles ou personnels). La description qu'elles font de leur exercice professionnel montre le souhait d'une prise en compte des situations particulières afin de créer les conditions de réussite de toutes les personnes, justifiant l'engagement de la direction et la recherche d'instauration d'une confiance réciproque. S'agissant du management des personnels, les directions soulignent unanimement leur préoccupation du bien-être de leurs collaborateurs, ressenti comme levier d'optimisation des conditions de travail, au bénéfice des élèves.

Dans cette perspective, les modalités managériales déclarées comme privilégiées se caractérisent par la veille et la vigilance dans une logique de soutien et de protection qui 
prend appui sur la valeur de l'exemplarité. Trois des directions interrogées estiment que la veille fait partie de leur mission. La Dir1 indique d'ailleurs être avant tout « veilleur du quotidien ", tâche qu'elle a aussi explicitement confiée à ses adjoints. Dans ce qu'elles expriment, il ne semble pas s'agir d'une posture de surveillance et de surplomb mais d'une attention réceptive qui demande disponibilité, écoute, observation pour identifier et pouvoir prendre en considération les fragilités des personnels. Les démarches qu'elles disent adopter sont avant tout d'accompagnement et de soutien. Les directions expriment souhaiter un management "en actes", dans une conception hiérarchique plus horizontale que verticale, de côte à côte plus que de face à face. Elles font aussi état de leur intention de démarches protectrices, y compris lors d'erreurs professionnelles. Sans faire jouer une solidarité corporatiste, elles expriment une volonté de dialogue et de compréhension, se fondant sur l'acceptation de l'erreur et le non-jugement. Les directions font également part de leur souci de valoriser les réussites et les points forts des personnels, qu'elles considèrent comme leviers de développement. Au travers des propos qu'elles tiennent sur leurs pratiques managériales, elles expriment le souci d'un "savoir être avec" visant à structurer les relations, arbitrer les conflits, mais aussi à motiver et impulser l'action collective. L'analyse montre également qu'à travers ces pratiques, les directions souhaitent faire preuve d'exemplarité, espérant la «contagion » (Dir.3) de leurs pratiques à celles des personnels.

36 Ainsi, l'analyse des données permet d'identifier que la bienveillance déclarée mise en œuvre dans le management par ces directions s'appuie sur ce qui paraît relever d'une réelle construction, permettant de déployer en situation un savoir agir complexe $\mathrm{e}^{13}$.

\section{La prise en compte des émotions}

Cette recherche ne tendait pas à prendre en compte les émotions des directions interrogées. Pourtant, à l'examen des données, on constate des propos saturés d'émotions négatives lorsqu'ils renvoient au versant administratif de la mission. Les directions ont alors toutes exprimé l'absence de bienveillance qu'ils ressentaient de la part de l'institution, envisagée au sens large, et le décalage par rapport à la réalité de leur terrain. On retrouve ici une mécanique proche, mais inversée, de la manifestation de la bienveillance dont ils témoignent quant à leurs pratiques managériales.

\subsection{La mobilisation de l'IE chez les personnels de direction}

\section{Compétences émotionnelles et éthique du care}

À l'analyse des REdEPP, nous relevons que ces directions sont attentives à l'émotivité de leur entourage professionnel, et ce, au-delà des tâches prescrites (Dejours, 2009). Leur représentation de la tâche réelle semble être ainsi liée à leurs valeurs, à leur profil de compétences, à leur expérience ou encore aux encadrements professionnels et légaux qui circonscrivent leurs responsabilités et leurs obligations professionnelles. Les propos analysés démontrent à maintes reprises la sollicitude et l'attention aux émotions des autres, et pour certains, envers leur propre personne. Le traitement des données montre que le "prendre soin" caractéristique du care converge vers deux nouvelles $\mathrm{CE}: 1$ ) prendre soin de son état émotionnel et 2) prendre soin de l'état émotionnel d'autrui. Le tableau 4 présente l'expression de ces deux nouvelles CE qui prennent appui sur l'éthique du care (Brugère, 2011; Tronto, 2009; Gilligan, 2008). Elles relèvent d'habiletés 
intrapersonnelles et interpersonnelles et complètent les modèles de Kotsou (2012, 2016) et de Mikolajczak et al. (2014) présentés au tableau 1.

Tableau 4 : Nouvelles compétences émotionnelles

\begin{tabular}{|c|c|}
\hline Prendre soin de son état émotionnel & Prendre soin de l'état émotionnel des autres \\
\hline Habiletés intrapersonnelles & Habiletés interpersonnelles \\
\hline $\begin{array}{l}\text { Avoir une attention à notre perception } \\
\text { émotionnelle, en termes de projection et } \\
\text { de protection de soi } \\
\text { - Habiletés (H) à anticiper les effets de } \\
\text { nos émotions en rapport aux événements } \\
\text { - H. à évaluer la charge émotionnelle } \\
\text { (l'impact) dans les situations } \\
\text { - H. à délibérer de cet état émotionnel } \\
\text { avec des ressources aidantes } \\
\text { - H. à maintenir un équilibre entre vie } \\
\text { professionnelle, personnelle et sociale }\end{array}$ & $\begin{array}{l}\text { Avoir une attention aux pratiques et aux récits des } \\
\text { autres, en termes de projection et de protection, } \\
\text { avec une certaine lecture empathique } \\
\text { - H. à percevoir et à répondre aux besoins } \\
\text { particuliers d'autrui en ayant un souci de l'autre } \\
\text { (Tronto, 2009) } \\
\text { - H. à anticiper les effets des émotions des autres } \\
\text { dans l'espace social } \\
\text { - H. à évaluer la charge émotionnelle (l'impact) dans } \\
\text { les situations } \\
\text { - H. à prendre en compte l'état de l'autre et } \\
\text { l'orienter vers un traitement de situation ou des } \\
\text { ressources aidantes }\end{array}$ \\
\hline
\end{tabular}

Un examen attentif des données a permis de mettre en évidence que ces compétences servent l'ajustement des pratiques de management, de manière à réduire certaines problématiques. Selon Abord de Châtillon et al. (2012), les problèmes de santé et de sécurité prennent de plus en plus d'importance aussi bien dans l'actualité et les médias que dans les préoccupations des directions. Il y a lieu de croire que l'exercice de ces deux nouvelles CE en management peut avoir un effet positif pour réduire ou amoindrir certains de ces problèmes en éducation et possiblement dans d'autres domaines professionnels.

\section{L'agir émotionnel en milieu d'éducation}

En complément à ces deux nouvelles définitions, Bélanger illustre un enrichissement du modèle de CE de Mikolajckak et al. (2014) dans le tableau 5 en présentant les quatre dimensions du modèle de $\mathrm{CE}$ et leurs caractéristiques, ainsi que la cinquième qui émerge des résultats de sa recherche. Cette dernière propose un regard différent, proche du care, et qui se manifeste par des comportements faisant preuve d'empathie envers soi et envers les autres. Nous observons une place dominante de «l'agir émotionnel » dans la pratique managériale de ces directions puisque les résultats d'analyse attestent de la mobilisation de $52,40 \%$ de CE contre $47,70 \%$ pour les compétences managériales exercées.

Tableau 5 : Modèle de compétences émotionnelles chez les directions : versant intrapersonnel et interpersonnel (Bélanger, 2017)

\begin{tabular}{|l|l|}
\hline Dimension & Caractéristiques \\
\hline
\end{tabular}




\begin{tabular}{|l|l|}
\hline $\begin{array}{l}\text { 1. Perception, évaluation et expression } \\
\text { des émotions }\end{array}$ & $\begin{array}{l}\text { Fonctionnement et discernement des émotions } \\
\text { Vocabulaire émotionnel } \\
\text { Expression du langage émotionnel (verbal ou non } \\
\text { verbal) }\end{array}$ \\
\hline $\begin{array}{l}\text { 2. Compréhension et analyse des } \\
\text { émotions }\end{array}$ & $\begin{array}{l}\text { Mentalisation ou états mentaux } \\
\text { Sens et intention perçus des comportements } \\
\text { Compréhension de leurs mécanismes, de leurs causes } \\
\text { et de leurs conséquences }\end{array}$ \\
\hline 3. Régulation réflexive des émotions & $\begin{array}{l}\text { Régulation des pensées } \\
\text { Réactions physiologiques } \\
\text { Lecture du comportement associé }\end{array}$ \\
\hline 4. Utilisation des émotions & $\begin{array}{l}\text { Connaissance du domaine des émotions } \\
\text { Compréhension de leurs mécanismes, de leurs causes } \\
\text { et de leurs conséquences }\end{array}$ \\
\hline Sollicitude et attention aux & $\begin{array}{l}\text { Relation de sollicitude à l'autre } \\
\text { Compréhension des manifestations émotionnelles } \\
\text { Impact et soutien des ressources d'aide }\end{array}$ \\
\hline
\end{tabular}

41 Des études ultérieures s'avèrent nécessaires pour permettre l'approfondissement de ce modèle de $\mathrm{CE}$ en milieu d'éducation. Il est primordial de s'interroger sur les effets, les enjeux personnels et socioprofessionnels des habiletés émotionnelles pour le développement des $\mathrm{CE}$.

\section{Discussion}

La mise en commun des résultats des deux recherches permet de proposer des éléments caractérisant ce qui pourrait relever d'un management bienveillant en éducation, en donnant une place à l'intelligence émotionnelle. Elle permet aussi de réinterroger ces pratiques au regard du modèle managérial contesté par les uns et plébiscité par les autres.

\subsection{Points de convergence entre les deux recherches}

Des points de convergence apparaissent et le croisement des travaux permet de dégager des éléments de caractérisation d'un agir managérial « bienveillant » à partir des discours des directions interrogées. Ces éléments sont présentés au tableau 6 et viennent enrichir les dimensions précitées.

Tableau 6 : Éléments de caractérisation de l'agir managérial bienveillant en éducation

\begin{tabular}{|l|l|l|}
\hline Dimensions & Contribution de la recherche de Réto & $\begin{array}{l}\text { Contribution de la recherche de } \\
\text { Bélanger }\end{array}$ \\
\hline
\end{tabular}




\begin{tabular}{|c|c|c|}
\hline $\begin{array}{l}1 . \\
\text { Intentionnelle }\end{array}$ & $\begin{array}{l}\text { Volonté et discernement du bien } \\
\text { prescrit: le bien commun de la } \\
\text { communauté éducative } \\
\text { Conscience incarnée de l'importance du } \\
\text { rôle confié (incarnation de l'autorité de } \\
\text { l'État) } \\
\text { Appui sur une intériorisation des } \\
\text { valeurs et des normes professionnelles } \\
\text { en lien avec la responsabilité } \\
\text { exercée (garantir les cadres de l'action } \\
\text { individuelle et collective) } \\
\text { Souci d'exemplarité pour une } \\
\text { contamination de la bienveillance }\end{array}$ & $\begin{array}{l}\text { Appui sur une culture } \\
\text { organisationnelle axée sur } \\
\text { l'affiliation et l'altruisme favorisant } \\
\text { la coopération, l'entraide, le } \\
\text { partage social, le soutien mutuel }\end{array}$ \\
\hline \multirow[t]{2}{*}{$\begin{array}{l}2 . \\
\text { Interactionnelle }\end{array}$} & $\begin{array}{l}\text { "Savoir être avec» dans la relation } \\
\text { managériale (accueil et hospitalité) } \\
\text { Exercice de l'autorité hiérarchique } \\
\text { horizontale, } \\
\text { d'accompagnement } \\
\text { Non-jugement et acceptation de } \\
\text { l'erreur de ses collaborateurs } \\
\begin{array}{l}\text { Appui sur une communication à la fois } \\
\text { franche et mesurée, permettant la } \\
\text { structuration de relations de confiance }\end{array}\end{array}$ & $\begin{array}{l}\text { Attention vigilante portée au climat } \\
\text { de coopération, d'entraide et de } \\
\text { partage social qui génère aussi } \\
\text { l'expression des CE entre les } \\
\text { personnes }\end{array}$ \\
\hline & \multicolumn{2}{|l|}{ Relation de sollicitude à l'autre } \\
\hline \multirow[t]{2}{*}{$\begin{array}{l}3 . \\
\text { Attentionnelle }\end{array}$} & $\begin{array}{l}\text { Exercice quotidien de veille et vigilance } \\
\text { Disponibilité, écoute, attention à } \\
\text { l'autre, à son contexte et à la situation } \\
\text { Prise en compte des situations } \\
\text { particulières, recherche de } \\
\text { compréhension } \\
\text { Désencombrement intérieur }\end{array}$ & $\begin{array}{l}\text { Mobilisation de l'attention pour la } \\
\text { perception, l'évaluation et } \\
\text { l'expression des émotions } \\
\text { Vigilance portée au climat } \\
\text { organisationnel }\end{array}$ \\
\hline & \multicolumn{2}{|l|}{ Reconnaissance et valorisation du travail } \\
\hline 4. Affective & $\begin{array}{l}\text { Prise en considération professionnelle } \\
\text { des affects et des émotions }\end{array}$ & $\begin{array}{l}\text { Compréhension, analyse et } \\
\text { régulation réflexive des émotions et } \\
\text { de leurs manifestations } \\
\text { Utilisation des émotions, adoption } \\
\text { d'un management } \\
\text { émotionnellement intelligent axé } \\
\text { sur l'affiliation (Kotsou, 2016) }\end{array}$ \\
\hline
\end{tabular}

Toutefois, notre échantillon restreint ainsi que l'appui sur une analyse secondaire des données ne permet, à ce stade, que de fournir quelques orientations concernant ce qui pourrait relever d'un construit théorique du management bienveillant. 


\subsection{Management bienveillant et intelligence émotionnelle}

Bélanger (2017) apporte un éclairage sur l'exercice des CE par les directions qui permet l'identification de leviers de formation pour le management des établissements scolaires. Montrant l'importance de la prise en compte de l'état émotionnel de soi et des autres, elle ouvre la voie à une meilleure prise en compte de l'IE et à une meilleure reconnaissance de son rôle dans le management. L'apport de Bélanger permet de développer la possibilité d'une réelle mise en travail des habiletés décrites au tableau 4. De plus, la mise en évidence de l'interdépendance existant entre le "prendre soin de son propre état émotionnel » et le " prendre soin de l'état émotionnel des autres » permet d'insister sur l'importance de la prise en compte de soi par la direction au travers des deux CE qui viennent compléter le modèle décrit au tableau 5 .

Finalement, au travers de ces deux CE, l'appel à prendre soin de soi par les directions permet de ne pas oublier la nécessité de bienveillance envers soi-même. En effet, face à l'intensité de la charge des responsabilités, face aux paradoxes d'un management bienveillant entre vouloir et pouvoir, projet SUR et projet AVEC, il peut être complexe pour ces personnels de trouver le juste point d'équilibre, de maintenir l'équilibre entre vie professionnelle, personnelle et sociale. Et le déséquilibre peut se révéler destructeur et produire une forte souffrance au travail. Or, la prise en compte de l'intelligence émotionnelle dans les pratiques managériales permet à la fois l'anticipation et la protection de soi et des autres en mobilisant l'habileté à évaluer la charge émotionnelle dans les situations professionnelles afin d'en anticiper les effets. Cette habileté permet aussi de susciter une régulation réflexive indispensable dans la gestion des situations complexes, lot quasi quotidien des directions. Or, Ferrière, Florin et Guimard (2016) recommandent la mise en œuvre de modalités de travail de gestion et de régulation émotionnelle dans les établissements scolaires. Par ailleurs, Bastien et Corbière (2018) mettent en lumière des pratiques louables au sein des organisations, mais qui sont strictement liées au travail et laissant de côté tout l'aspect relationnel, notamment la relation avec le superviseur immédiat et les collègues de travail. Ils mentionnent l'importance du superviseur à vouloir mettre en place des accommodements de travail, de son attitude, de son soutien et de son empathie auprès de l'employé qui effectue un retour au travail après une période d'épuisement professionnel ou de dépression. Dans le même ordre d'idées, Marchand et Beauregard (2012) mentionnent l'importance à accorder à l'épuisement professionnel, c'est à dire l'épuisement émotionnel (qui découle d'un surcroît d'effort, et aboutit sur du cynisme et une diminution de l'efficacité au travail). Ils ont "remarqué que l'épuisement (ou abattement) émotionnel est la composante qui s'avère la plus déterminante dans l'apparition de l'épuisement professionnel au fil du temps » (p. 1). Dans un tel contexte, l'un des défis qu'ont à relever les directions d'établissements scolaires est de mettre en place de bonnes conditions de travail, voire des accommodements permettant à leurs personnels de travailler en respect de certaines capacités, et ce, dans une culture de bienveillance éducative.

\subsection{Entre suspicion et fascination, quelle déclinaison du management bienveillant pour les établissements scolaires?} qui tend à s'imposer comme un modèle possible, mais aussi les attaques qu'il suscite. Les 
réserves posées par Linhart (2015) sont à prendre en considération afin d'éviter les conduites manipulatoires sous couvert du masque des bonnes intentions. Toutefois, il nous semble que les logiques et modalités qu'elle décrit sont éloignées de celles qui apparaissent dans les propos des directions interrogées. Certes, il ne s'agit que de déclaratif, ce qui doit pousser à la vigilance, mais les propos tenus par les personnes interrogées, loin de toute arrogance, témoignent d'un souhait de compréhension des personnels, de la reconnaissance de la complexité de leur travail et d'un soutien face à leur exercice professionnel difficile. Leur mode de management vise à s'inscrire dans un leadership partagé avec un élargissement du pouvoir d'action des différents acteurs et à valoriser les formes de travail collaboratif.

Au-delà de la dimension des personnes, ces caractéristiques que nous avons relevées s'expliquent sans doute aussi par les spécificités du management des établissements scolaires : l'ensemble de l'activité des personnels est finalisé par les missions assignées à l'école et les logiques économiques et de productivité y diffèrent donc des autres organisations. De plus, les établissements scolaires sont des organisations faiblement hiérarchisées, avec des groupes de personnels relativement homogènes: la mise en concurrence des salariés y est faible, la longévité dans l'emploi importante, la stabilité dans les fonctions forte. Dans le contexte des établissements scolaires français, au final, l'enjeu managérial prioritaire n'est d'ailleurs pas de passer de la collectivisation à l'individualisation mais plutôt de parvenir à favoriser le développement d'une identité professionnelle collective et à développer les dynamiques d'un leadership partagé.

49 Au final, au travers de la caractérisation proposée, il est davantage question ici, pour les directions interrogées, de ménagement et d'aménagement de la relation de travail plus que de management de type entrepreneurial. Il s'agit moins de promouvoir un mouvement managérial qui laisse voir des limites que de proposer une réflexion sur les compétences et la posture développées par les directions pour agir, du mieux qu'elles peuvent, dans les contextes complexes qui sont les leurs.

\section{Conclusion}

Le rapprochement des recherches a permis d'ouvrir la réflexion à une approche du management des établissements scolaires, en réponse aux préoccupations sociales et scientifiques françaises et québécoises. L'analyse des données collectées montre que la responsabilité confiée aux directions est l'occasion de mettre en œuvre un agir managérial qui s'appuie sur le "prendre soin » des personnels et renvoie aux dimensions de la bienveillance. Si cette dernière n'apparaît pas dans les prescriptions professionnelles des directions, on peut conclure que sa présence peut être considérée par les directions interrogées comme porteuse et productive et qu'elle ne relève pas de la seule qualité personnelle. La place donnée à la prise en compte des émotions dans l'agir managérial bienveillant permet d'enrichir l'approche et contribue à montrer qu'il s'agit d'une dimension professionnelle construite et structurée, s'appuyant sur le cadre de référence des personnes et s'exprimant au travers d'habiletés interpersonnelles comme intrapersonnelles dans les situations complexes. Loin de la stratégie manipulatoire, elle permet aussi d'envisager des pistes de protection qui faciliteraient, pour les directions, la conjugaison de l'exercice, leur responsabilité et leur propre bien-être au travail. Ainsi, accorder une attention spécifique aux acteurs clés que sont les directions constituerait sans doute un levier efficace d'amélioration du système. Des perspectives sont ouvertes 
pour les accompagner dans le développement des compétences managériales spécifiques. En effet, il s'agit de réfléchir autrement la formation de ces dirigeants qui sont soumis à une forte exigence. Selon Dejours (2012), « il faut parvenir à faire évoluer les critères et les méthodes dans les écoles de management, afin de prendre en compte les éléments humains qui participent à l'accomplissement du travail»(p.37). Cela ouvre à de nouvelles voies de formation qui devront tenir compte du contexte spécifique de l'institution scolaire.

\section{BIBLIOGRAPHIE}

Abord de Châtillon, E., Bachelard, O. \& Carpentier, S. (2012). Risques psychosociaux, santé et sécurité au travail : une perspective managériale. Paris : Vuibert.

Aubert, N. (2003). Le culte de l'urgence : la société malade du temps. Paris : Flammarion.

Bastien, M.-F. \& Corbière, M. (2018). Return-to-Work Following Depression: What Work Accommodations Do Employers and Human Resources Directors Put in Place? Journal of Occupational Rehabilitation, 1-10.

Bélanger, L. (2016). Les compétences émotionnelles : un facteur de vulnérabilité des gestionnaires du réseau collégial québécois. Dans B. Raveleau et F. Ben Hassel (dir.), Gérer la vulnérabilité et la résilience en milieu professionnel (pp. 151-170). Paris : L'Harmattan.

Bélanger, L. (2017). Les compétences émotionnelles exercées en situation professionnelle par les directrices et les directeurs généraux des cégeps au Québec. (Thèse de doctorat en éducation). Sherbrooke : Faculté d'éducation, Université de Sherbrooke. Doctorat Éducation, Carriérologie et Éthique. Angers : Université catholique de l'Ouest.

Bertaux, D. (2010). Le récit de vie: L'enquête et ses méthodes. Paris : Armand Colin.

Brugère, F. (2011). L'éthique du care. Paris : PUF.

Debarbieux, E. (2015). Du « climat scolaire » : définitions, effets et politiques publiques. Éducation \& Formations (F. Murat et C. Simonis-Sueur (dir.), « Climat scolaire et bien-être à l'école »), 88-89. Paris : Ministère de l'Éducation nationale, de l'Enseignement supérieur et de la Recherche.

Dutercq, Y., Gather Thurler, M. \& Pelletier, G. (dir.) (2015). Le leadership éducatif : Entre défi et fiction . Louvain-la-Neuve, Belgique : De Boeck Supérieur.

Dutercq, Y. \& Mons, N. (2015). Chapitre 1. Les chefs d'établissement français : un leadership sous le signe du marketing ? Dans Y. Dutercq, M. Gather Thurler \& G. Pelletier (dir.), Le leadership éducatif : Entre défi et fiction (pp. 21-36). Louvain-la-Neuve, Belgique : De Boeck Supérieur.

Dejours, C. (2009). Travail vivant. 2 : Travail et émancipation. Paris : Payot.

Dejours, C. (2015). Le choix: souffrir au travail n'est pas une fatalité. Montrouge : Bayard.

Endrizzi, L. \& Thibert, R. (2012). Quels leaderships pour la réussite de tous les élèves ? Dossier d'actualité Veille et Analyses, 73, avril. Repéré à http://ife.ens-lyon.fr/vst/DA-Veille/73avril-2012.pdf (Consulté le 26 aout 2017). 
Ehrenberg, A. (1998). La fatigue d'être soi : dépression et société. Paris : Odile Jacob.

Ferrière, S., Florin, A. \& Guimard, P. (2016). Les interactions entre les acteurs pour la qualité de vie dans les établissements scolaires. Paris : Cnesco.

Foulquié, P. \& Saint-Jean, R. (1969). Dictionnaire de la langue philosophique (2 éd.). Paris : PUF.

Garon, R., Théorêt, M., Hrimech, M. \& Carpentier, A. (2006). Résilience et vulnérabilité chez des chefs d'établissement scolaire : une étude exploratoire. Psychologie du travail et des organisations, 12 , 327-337.

Gaulejac, V. de. (2004). Le sujet manqué. Dans N. Aubert (dir.), L'individu hypermoderne (pp. 129-143). Toulouse : Érès.

Gilligan, C. (2008). Une voix différente : pour une éthique du care. Paris : Flammarion.

Gouvernement du Québec (2013). Guide explicatif et questionnaire d'analyse. Évaluation d'emploi personnel d'encadrement collèges d'enseignement général et professionnel. Québec: Ministère de l'Enseignement supérieur, de la Recherche, de la Science et de la Technologie, Direction générale des relations du travail.

Gouvernement du Québec (2016). Loi sur les collèges d'enseignement général et professionnel. Repéré à http://legisquebec.gouv.qc.ca/fr/ShowDoc/cs/C-29 (Consulté le 28 février 2017).

Kotsou, I. (2012). Intelligence émotionnelle et management : comprendre et utiliser la force des émotions

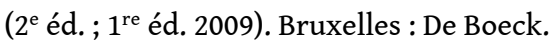

Kotsou, I. (2016). Intelligence émotionnelle et management : comprendre et utiliser la force des émotions

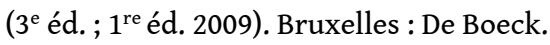

Lafranchise, N., Lafortune, L. \& Rousseau, N. (2013). Prise en compte des émotions, compétence émotionnelle et accompagnement socioconstructiviste en insertion professionnelle. Dans J. Pharand et M. Doucet (dir.), En éducation, quand les émotions s'en mêlent! (pp. 146-173). Québec : Presses de l'université du Québec.

Lapeyronnie, D. (2014). Pour une école innovante. Synthèse des travaux du Conseil national pour l'innovation pour la réussite éducative. Repéré à http://cache.media.education.gouv.fr/ file/2014/81/1/CNIRE-Rapport-Pour-une-ecole-innovante_365811.pdf.

Laugier, S. \& Paperman, P. (2008). La voix différente et les éthiques du care. Dans C. Gilligan, Une voix différente, pour une éthique du care. Paris : Flammarion.

Le Boterf, G. (2010). Repenser la compétence. Pour dépasser les idées reçues : quinze propositions ( $2^{\mathrm{e} e ́ d . ~}$

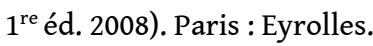

Le Boterf, G. (2013). Construire les compétences individuelles et collectives. Le modèle : agir avec compétence en situation, les réponses à plus de 100 questions ( $1^{\text {re }}$ éd. 2000). Paris : Eyrolles.

Letor, C. (2006). Reconnaissance des compétences émotionnelles comme compétences professionnelles : le cas des enseignants. Analyse des représentations sociales d'acteurs pédagogiques. Les cahiers de recherche en éducation et formation, 53, 1-38.

Linhart, D. (2015). La comédie humaine du travail : de la déshumanisation taylorienne à la surhumanisation managériale. Paris : Érès, coll. « Sociologie clinique ».

Marchand, A. \& Beauregard, N. (2018). Épuisement professionnel : les symptômes varient grandement selon les périodes de vie. Montréal : Université de Montréal. 
Mayer, J. D. et Salovey, P. (1997). What is Emotional Intelligence? Dans P. Salovey \& D. J. Sluyter (éds.), Emotional development and emotional intelligence: Implications for educators (pp. 3-31). New York : Basic Books.

MEN (2013). Loi d'orientation et de programmation pour la refondation de l'École de la République (2013-595 - 8/07/2013). Paris : Ministère de l'Éducation nationale, de l'Enseignement supérieur et de la Recherche.

Mikolajczak, M., Quoidbach, J., Kotsou, I. \& Nélis, D. (2014). Les compétences émotionnelles. ( $2^{\mathrm{e}}$ éd. ; 1 re éd. 2009) Paris : Dunod.

Mintzberg, H. (1998). Structure et dynamique des organisations ( $3^{\mathrm{e}}$ éd.). Paris : Organisation.

Mintzberg, H. (2010). Gérer. Montréal : Transcontinental.

Mintzberg, H. (2014). Manager l'essentiel. Ce que font vraiment les managers... et ce qu'ils pourraient faire mieux. Paris : Magnard-Vuibert.

Morin, E. (2005). Introduction à la pensée complexe. Paris : Seuil.

Murat, F. \& Simonis-Sueur, C. (2015). Climat scolaire et bien-être à l'école. Education \& Formations, 88-89. Paris : Ministère de l'Éducation nationale, de l'Enseignement supérieur et de la Recherche.

OCDE (2008). Améliorer la direction des établissements scolaires, volume 1 : Politiques et pratiques. OCDE. Repéré à http://www.oecd.org/fr/edu/scolaire/44374906.pdf (Consulté le 2 février 2016).

OCDE (2012). Introduction. Dans Des compétences meilleures pour des emplois meilleurs et une vie meilleure. Une approche stratégique des politiques sur les compétences. OCDE. DOI : http:// dx.doi.org/10.1787/9789264178717-2-fr.

OCDE (2014). Principaux résultats de l'Enquête PISA 2012. Ce que les élèves de 15 ans savent et ce qu'ils peuvent faire avec ce qu'ils savent. Repéré à https://www.oecd.org/pisa/keyfindings/pisa-2012results-overview-FR.pdf (Consulté le 26 août 2017).

Paillé, P. (1994). L'analyse par théorisation ancrée. Cahiers de recherche sociologique, 23, 147-181.

Paillé, P. \& Mucchielli, A. (2013). L'analyse qualitative en sciences humaines et sociales ( $3^{\mathrm{e}}$ éd. ; $1^{\mathrm{re}}$ éd. 2002). Paris : Armand Colin.

Pharand, J. \& Doucet, M. (2013). En éducation, quand les émotions s'en mêlent. Enseignement, apprentissage et accompagnement. Québec : Presses de l'université du Québec.

Poirel, E. (2012). Pour de nouvelles compétences professionnelles chez les gestionnaires en administration de l'éducation : les compétences émotionnelles. Dans Actes du Colloque ADERAE 2012 (pp. 32-44). Montréal.

Prairat, E. (2012). La responsabilité. Le Télémaque, 2(42), 19-34.

Rascle, N., Bergugnat, L., Florin, A. \& Guimard, P. (2016). Qualité de vie des enseignants en relation avec celle des élèves : revue de question, recommandations. Paris : Cnesco.

Réto, G. (2016). Le caring, une voie pour reconfigurer l'École française au moment de sa refondation ? Éducation et socialisation, 40. Repéré à http://edso.revues.org/1504 (Consulté le 11 mars 2017).

Réto, G. (2018a). La bienveillance dans le champ scolaire. Caractérisation des pratiques et actualisation selon des membres du personnel enseignant de collège, des chefs d'établissement et des experts du monde de l'éducation. (Thèse de doctorat en éducation). Sherbrooke : Faculté d'éducation, Université de Sherbrooke. Doctorat Éducation, Carriérologie et Éthique. Angers : Université catholique de l'Ouest. 
Réto, G. (2018b). La bienveillance à l'École : vers un changement de paradigme. Recherches et Éducations, 17 («Le Bien-être à l'école : un processus de production du bien-être ?»). Sous presse.

Rynes, S., Bartunek, J., Dutton, J \& Margolis, J. (2012). Care and Compassion Through an Organizational Lens: Opening Up New Possibilities. Academy of Management Review 2012, 4(37) (« Understanding and creating caring and compassionate organizations »), 503-523.

Salovey, P. \& Mayer, J. D. (1990). Emotional intelligence, imagination, cognition and personality, Emotional Intelligence, 9, 185-211.

Tardif, J., Fortier, G. \& Préfontaine, C. (2006). L'évaluation des compétences : documenter le parcours de développement. Montréal : Chenelière éducation.

Tronto, J. C. (2009). Un monde vulnérable : pour une politique du care. Paris : Éditions La Découverte. Vermersch, P. (2014). L'entretien d'explicitation ( $2^{\mathrm{e}}$ éd.). Issy-les-Moulineaux : ESF éditeur.

Vienneau, R. (2005). Apprentissage et enseignement, théories et pratiques. Montréal, Canada : Gaétan Morin éditeur.

Wacheux, F. (1996). Méthodes qualitatives et recherche en gestion. Paris : Economica.

Zapata, A. (2004). L'épistémologie des pratiques pour l'unité du savoir. Paris : L'Harmattan.

Zielinski, A. (2011). La vulnérabilité dans la relation de soin. Cahiers philosophiques, 2(125), 89-106.

\section{NOTES}

1. Le personnel de direction de ces établissements est désigné différemment selon les pays (direction générale au Québec, chef d'établissement, principal ou directeur en France). Pour faciliter la compréhension de cet article, nous emploierons «direction» pour désigner la dirigeante ou le dirigeant de l'établissement.

2. Le terme vient de "mesnager ", emprunté au domaine de l'équitation du XVI ${ }^{\mathrm{e}}$ siècle, et signifie "tenir les rênes» (Mintzberg, 2014, p. 9). Par cooccurrence lexicale, le terme "manager» renvoie à la fonction d'un cadre dirigeant d'une organisation ou au verbe " manager ou gérer » qui traduit les actions de gestion effectuées par la personne dirigeante en situation de management.

3. Le réseau collégial public est la somme de tous les cégeps (études postsecondaires en enseignement supérieur) du secteur public et se compose de 48 collèges : 5 cégeps de langue anglaise, 43 de langue française, 2 collèges régionaux.

4. Le terme "manager » renvoie à la fonction d'un cadre dirigeant d'une organisation ou au verbe «manager» ou "gérer» qui traduit les actions de gestion effectuées par la personne dirigeante en situation de management.

5. Afin de maintenir la cohérence de mise en dialogue entre nos deux recherches, nous avons été tenues de circonscrire notre approche mais nous sommes conscientes qu'elle limite la perspective offerte par le care.

6. Traduction libre : L'intelligence émotionnelle implique la capacité à percevoir avec exactitude, évaluer et exprimer l'émotion; la capacité d'accéder et/ou de générer des sentiments quand ils facilitent la pensée ; la capacité de comprendre l'émotion et la connaissance émotionnelle; et la capacité de réguler les émotions pour favoriser la croissance émotionnelle et intellectuelle.

7. Selon Zapata (2004), la pratique professionnelle possède les caractéristiques suivantes : elle est contextuelle, du fait qu'elle existe au sein d'un contexte, avec des conditions adaptées à la nature de la pratique, permettant d'appréhender le sens de l'action; elle est événementielle, car elle ne se situe pas dans une continuité d'actions, mais fait plutôt référence à une suite de moments bien 
repérables, en rupture avec d'autres moments ou activités; elle est singulière, car elle ne peut jamais se reproduire de manière identique et ne peut être transposable d'une situation à une autre. Ainsi, peut-on définir le contexte professionnel ou encore le contexte situé.

8. Les types d'établissements retenus ne sont pas des institutions du même ordre d'enseignement. Toutefois, il existe de grandes similitudes sur la structure et la dynamique des établissements. Par ailleurs, même si les structures des organisations sont variées et dépendantes de leurs environnements, il s'en dégage certains types de configuration structurelle, soit des idéaux types ou des catégories distinctes puis certaines régularités dynamiques liées à ces types de configuration (Mintzberg, 1998).

9. Leurs missions et caractéristiques sont présentées en section 1.2.

10. Le terme urbain correspond au statut municipal selon la région administrative. Le terme rural ou régional s'appuie de la Loi sur les collèges d'enseignement général et professionnel. Gouvernement du Québec (2016). Loi sur les collèges d'enseignement général et professionnel. Repéré à http://legisquebec.gouv.qc.ca/fr/ShowDoc/cs/C-29 (Consulté le 28 février 2017).

11. Des entretiens ont été menés avec d'autres catégories de personnels mais seules les données recueillies auprès des directions sont utilisées ici. De plus, seules les parties des entretiens questionnant la potentialité d'un exercice « bienveillant » de la responsabilité sont mobilisées ici. 12. Cela n'a d'ailleurs pas été démenti par le personnel enseignant également rencontré en entrevue.

13. Cette recherche se limite au déclaratif des directions, sans prendre en compte l'observation des pratiques, ce qui peut générer de la suspicion quant à une réelle mise en œuvre de ces pratiques. Toutefois, des entrevues menées avec des personnels de trois des quatre établissements concernés et les données collectées dans ce cadre n'ont pas contredit ou nuancé ces déclarations.

\section{RÉSUMÉS}

Cet article propose d'articuler la question de la bienveillance avec celle de l'intelligence émotionnelle dans le cadre du management des établissements scolaires. Il met en dialogue deux recherches (Bélanger, 2017; Réto, 2017) qui s'intéressent à un échantillon de dirigeants d'établissement d'enseignement en contexte québécois et en contexte français. Il prend appui sur la clarification de la notion de bienveillance, en lien avec l'éthique du care, et précise l'interdépendance de l'intelligence émotionnelle et des compétences émotionnelles en lien avec le modèle du savoir agir avec compétence. L'étude sur les compétences émotionnelles exercées en situation professionnelle par les directrices et les directeurs généraux des cégeps au Québec a permis d'enrichir le modèle de Mikolajczak et al. (2014) par l'ajout de deux nouvelles compétences inspirées par l'éthique du care. Cette nouvelle dimension conceptuelle suscite une réflexion associative entre l'intelligence émotionnelle et la bienveillance. Un regard est donc porté sur le «management bienveillant»et permet d'interroger le rôle de l'intelligence émotionnelle dans sa mise en pratique par les directions d'établissement scolaire.

This article suggest to articulate the question of caring and the issue of emotional intelligence within the School Management framework. It open a dialogue between two researches (Bélanger, 2017, Réto, 2017) which have studied in a sample of leaders of educational institutions in Quebec and in France. It builds on the clarification of the notion of caring, in relation to the ethics of 
care, and clarifies the interrelationship of emotional intelligence and emotional skills within the framework of knowledge competently. The study on the emotional competences exercised in a professional situation by the directors-general of cegeps in Quebec allowed to improve the model of Mikolajczak et al. (2014) by adding two new skills inspired by the ethics of care. This new conceptual dimension triggers an associative reflection between emotional intelligence and caring. A look is thus made on "caring management" and makes it possible to question the role of emotional intelligence in its implementation by the school management.

\section{INDEX}

Keywords : caring, emotional intelligence, emotional skill, caring management, education Mots-clés : bienveillance, intelligence émotionnelle, compétence émotionnelle, management bienveillant, éducation

\section{AUTEURS}

\section{LYNE BÉLANGER}

Ph. D. en Éducation à l'Université de Sherbrooke (Canada) ; Doctorat en Éducation, Carriérologie et Éthique à l'Université catholique de l'Ouest à Angers (France)

\section{GWÉNOLA RÉTO}

Ph. D. en Éducation à l'Université de Sherbrooke (Canada) ; Doctorat en Éducation, Carriérologie et Éthique à l'Université catholique de l'Ouest à Angers (France) 